\title{
Síndrome de Quemado (Burnout) en Docentes Universitarios: El Caso de un Centro de Estudios del Caribe Colombiano
}

\author{
Elías A. Bedoya ${ }^{(1)^{*}}$, Nancy E. Vega ${ }^{(2)}$, Carlos A. Severiche ${ }^{(2)}$, María J. Meza ${ }^{(2)}$ \\ (1) Fundación Universitaria Tecnológico Comfenalco, Coordinador de investigación del Programa de \\ Tecnología en Seguridad e Higiene Ocupacional, Grupo de Investigación CIPTEC, Cartagena de Indias, \\ Bolívar-Colombia (e-mail: ebedoya@tecnologicocomfenalco.edu.co). \\ (2) Fundación Universitaria Tecnológico Comfenalco, Programa de Tecnología en Seguridad e Higiene \\ Ocupacional. Cartagena de Indias, Bolívar-Colombia
}

${ }^{*}$ Autor a quien debe ser dirigida la correspondencia

Recibido Abr. 7, 2017; Aceptado Jun. 13, 2017; Versión final Ago. 8, 2017, Publicado Dic. 2017

\begin{abstract}
Resumen
Se evaluó el síndrome de quemado (burnout) y factores asociados en docentes de una institución superior del caribe colombiano. El estudio es analítico de corte transversal. La población la constituyen 150 docentes de una institución superior pública. La recolección de información fue por medio de encuesta sociodemográfica y el instrumento Maslach Burnout Inventory (MBI). Para el análisis estadístico se utilizó el programa SPSS versión $20 \circledast$, aplicando las pruebas no paramétricas $U$ de Mann-Whitney y Kruskall Wallis para estimar asociaciones entre variables. Los resultados muestran que la realización personal y los síntomas de estrés se encuentran asociados al género. Los dos fueron más altos en el género femenino. Los docentes que cuentan con solo pregrado y con menor antigüedad reportan mayores puntajes en agotamiento emocional. Se concluye que el agotamiento emocional varía significativamente según área de desempeño de los profesores.
\end{abstract} quemado

\section{Burnout Syndrome in University Teachers: the Case of a Study Center in the Colombian Caribbean}

\begin{abstract}
Burnout syndrome and associated factors were evaluated in teachers of a Colombian higher education institution. The study is analytical of cross-sectional type. The population consists of 150 teachers from a public higher education institution. The data collection was done by means of a sociodemographic survey and the Maslach Burnout Inventory (MBI). For the statistical analysis, the SPSS version $20{ }^{\circledR}$ program was used, applying the non-parametric $U$ tests of Mann-Whitney and Kruskall Wallis to estimate associations between variables. The results show that personal fulfillment and stress symptoms are associated with gender. Both were higher for the female gender. Teachers with only undergraduate and lower seniors report higher scores on emotional stress. It is concluded that emotional stress varies significantly according to performance area of the teachers.
\end{abstract}

Keywords: emotional stress; university teachers; educational practices; burnout syndrome 


\section{INTRODUCCIÓN}

El estrés y las enfermedades psicosomáticas actualmente son más frecuentes y afectan la salud de los individuos, así como su rendimiento profesional (Malinen y Savolainen, 2016). El Síndrome de Burnout, también conocido como "síndrome del estrés crónico laboral", "síndrome de desgaste profesional", "síndrome del quemado" o "síndrome del estrés laboral asistencial", es un estado de cansancio físico y emocional típico de aquellos individuos que ejercen profesiones de ayuda a otros individuos y que han sido elegidas libremente. Este síndrome se caracteriza por actitudes negativas hacia los sujetos que constituyen el objeto de su trabajo y hacia el propio trabajo (Borges et al., 2012; Barutçu y Serinkan, 2013). Maslach y Jackson (1981), lo definen como un síndrome que consta básicamente de tres dimensiones que son: el agotamiento emocional, la despersonalización o fase de desarrollo de actitudes y respuestas negativas hacia los beneficiarios de su trabajo y la falta de realización personal o fase donde el trabajador siente que las demandas de su trabajo exceden sus capacidades de respuestas y la frustración es el resultado final de una carrera de muchos años que comenzó con vocación y entusiasmo en el servicio a los otros.

Es reconocido entre los docentes el carácter estresante de su profesión, creencia avalada científicamente por los especialistas en el tema de la salud del trabajador. Los maestros presentan perfiles epidemiológicos que los distinguen de otros profesionales, incluso de los servicios, como ellos. Las patologías más frecuentes son las alteraciones circulatorias, del aparato fonológico y los trastornos de la salud mental, en especial la ansiedad y la depresión. La idea de que la profesión docente constituye un riesgo para desarrollar trastornos psiquiátricos, es un tema de discusión (Oramas et al., 2007; Arquero y Donoso, 2013). Esta interacción, situada en un determinado entorno: la escuela, con sus peculiaridades y complejidades pueden generar sentimientos ambiguos y respuestas contradictorias y frustrantes. Se trata de un trastorno adaptativo asociado a un inadecuado afrontamiento de las demandas psicológicas del trabajo que daña, o perjudica la calidad de vida de las personas que lo padece, disminuye la respuesta personal y repercute negativamente en la calidad de la enseñanza (Botero, 2012; Fiorilli et al., 2015).

Entre los estudios que se relacionan con la temática expuesta, pueden mencionarse, un estudio en docentes de ciencias básicas en Guadalajara-México, donde se encontraron altos niveles de cansancio emocional (25,9\%), baja realización personal en el trabajo (21,6\%) y despersonalización (5,6\%). En Colombia, el tema del síndrome de Burnout se ha investigado relativamente poco. Sin embargo, se cuentan con investigaciones sobre el tema, como es el caso de un estudio realizado en la ciudad de Medellín, evidenciaron que $36,8,33,4$ y $5,4 \%$ de los docentes, refirieron cansancio emocional, despersonalización y baja realización personal, respectivamente (Rojas et al., 2009). Por lo anterior, el presente estudio se propone identificar los factores asociados al síndrome de Burnout y la prevalencia del mismo en docentes de una institución superior pública de Cartagena de indias, en el caribe colombiano.

En el estudio desarrollado por Andina (2015) titulado como: "El Burnout de los profesores en relación con su inteligencia emocional y rasgos de la personalidad" se indica sobre la inteligencia emocional de los profesores está correlacionada negativamente con el agotamiento de los maestros, lo que establece que entre mejor se manejen los educadores pueden reducir el agotamiento y elevar el nivel de participación de los estudiantes en la dinámica de aprendizaje que garantice su éxito tanto personal como profesional, para esto se precisa sobre la necesidad de formular programas para docentes con asistencia psicopedagógica que ayude a prevenir situaciones como el quemarse en el trabajo. En estudios similares sobre el desgaste y agotamiento en docentes, para este caso la investigación de Kuimova et al., (2015) demuestra que el rendimiento de la educación es diferente a la forma en que funciona un negocio, y por esto las condiciones personales indefendibles que están fuera del control de los maestros que al estar presente en al ámbito de trabajo de manera prolongada causan agotamiento emocional e Insatisfacción, que además puede desalentar la confianza de los maestros y conducir a una mala salud física, (Vysotskaya et al., 2015) acompañado por sensaciones como el desgaste, disminución de la eficiencia en el trabajo y otros malestares y para mejorar tal condición se propone una política de personal equitativa, posibilidad de ascenso profesional, liderazgo efectivo y administración para el diálogo, la disponibilidad de normas generalmente aceptadas por los docentes.

Situaciones como la satisfacción con la vida, la salud y el agotamiento (agotamiento emocional, despersonalización o cinismo y logro) son eventos que se han documentado. Es interesante tener en cuenta que el disfrutar el trabajando, también tiene relación con aspectos como el horario, planificación y organización del trabajo, que manejado adecuadamente se denomina como la dimensión positiva del bienestar (Suárez, 2017). Síntomas como circunstancias psicopatémicas, insatisfacción y el "estado oprimido" junto a la ansiedad y la depresión, son reacciones emocionales dentro de los trastornos psicosomáticos y psico-vegetativos relacionados con la aparición de agotamiento entre los profesores afectados por el síndrome. Cazan y Năstasă (2015) han precisado que relaciones significativas entre la inteligencia emocional, el agotamiento y la satisfacción con la vida son demostradas como reflejos del 
agotamiento académico resultados de la conjugación de variables rutinarias mencionadas por el estudio de Barutçu \& Serinkan (2013) Donde afectantes en los grupos docentes-estudiantes objeto de evaluaciones donde la Inteligencia emocional el diagnóstico del burnout y satisfacción con la vida fueron apreciadas.

Por otro lado, reflexiones en la predicción del estrés en el aula y el agotamiento se reportan como resultados mostraron que estas dos características personales se relacionan con la tensión de los maestros en formas únicas y distintivas. Se vaticinó sobre predictores positivos del estrés y el agotamiento, representando una proporción significativa de la varianza explicada en ambos criterios más allá de las características percibidas del trabajo. La reflexión de Košir, et al., (2015) no predijo el estrés y el agotamiento, pero determinaron relaciones entre las características percibidas del trabajo y el estrés causada por la carga de trabajo, autonomía y estrés claramente vivenciadas en los docentes fijando una la relación negativa entre el apoyo al compañero y el estrés. Lo anterior puede indicar que maestros pueden verse influenciados por condiciones del medio ambiente y por el afrontamiento ante distintas exigencias que preceden al agotamiento o diversas combinaciones los factores anteriormente mencionados, lo que sugiere la necesidad de un enfoque amplio para abordar la problemática (Foley y Murphy 2015).

La afectación en profesores está determinada sólo por factores de personalidad, mientras que el agotamiento y el cinismo están determinados tanto por variables individuales como organizacionales. Los maestros que experimentan burnout perciben un mayor nivel de desajuste entre ellos mismos y el ambiente de trabajo, en comparación con los maestros comprometidos demostrando una mejor alineación. Los profesores comprometidos eran más bajos en la afectividad negativa y más altos en la auto-dirección en comparación con el grupo de burnout, los factores individuales en el desarrollo del agotamiento y el compromiso de los maestros (Mojsa et al., 2015).

Autores como De Caroli y Sagone (2013) en su estudio sobre representación profesional el riesgo de burnout en Maestros, analizaron la relación entre los niveles de burnout y las diversas maneras que estos se alcanzan a afectar profesionalmente, revelando correlaciones negativas entre el autoconcepto profesional y el agotamiento. Se han analizado las diferencias significativas por sexo y años de enseñanza, notando que las profesoras experimentan mayores niveles de agotamiento emocional que los profesores varones, sin diferencias significativas en otras dimensiones. Estudios en grupos heterogéneos de docentes adelantado por Hozo et al., (2015) puntualizan que controles médicos regulares y acciones específicas que hacen prevención específica del síndrome de burnout para evitar su desarrollo ulterior. Maestros consideran diferentes factores como la principal causa de burnout entre actividades de planeación, toma de decisiones y dificultades en la interacción-cooperación entre grupos de trabajo académico que difieren de la relación entre docentes y estudiantes como los afirman estudios otros estudios al referirse a las causas de desgaste entre los maestros (Khezerlou, 2013).

\section{MATERIALES Y METODOS}

Se realizó un estudio de corte transversal. La población estuvo conformada por 150 docentes de una institución superior pública de Cartagena de Indias, caribe colombiano. Se aplicó una encuesta sociodemográfica para recolectar información básica de los participantes. Además, se utilizó el instrumento Maslach Burnout Inventory (MBI) de Maslach y Jackson en el que se plantea al sujeto una serie de enunciados sobre los sentimientos y pensamientos con relación a su interacción con el trabajo.

Está formado por 22 ítems que se valoran con una escala tipo Likert, mediante un rango de siete adjetivos que van de "nunca" a "todos los días", con qué frecuencia experimenta cada una de las situaciones descritas en los ítems. El cuestionario tiene tres factores: 1) La subescala de agotamiento emocional (Emotional Exhaustion - EE) está formada por nueve ítems que describen sentimientos de estar saturado y cansado emocionalmente por el trabajo. Se estima como puntuación máxima 54 puntos, Donde puntuar 15 ó menos es valor bajo, de 15 a 24 medio y más de 24 es puntaje alto. 2) La subescala de despersonalización (Despersonalization - D) está formada por cinco ítems que describen una respuesta fría e impersonal y falta de sentimientos e insensibilidad hacia los sujetos objeto de atención, la puntuación máxima de esta subescala es 30 puntos, identificados puntajes como 4 ó menos es valor bajo, entre 4 y 9 medio y más de 9 alto. 3) La subescala de realización personal en el trabajo (Personal Accomplishment PA) está compuesta por ocho ítems que describen sentimientos de competencia y eficacia en el trabajo, tendencia a evaluar el propio trabajo de forma negativa y vivencia de insuficiencia profesional, funciona en sentido contrario a las anteriores, con un puntaje máximo de 48 puntos donde de 0 a 30 puntos indicaría baja realización personal, de 34 a 39 intermedia y superior a 40, sensación de logro. Porque en este caso la puntuación es inversamente proporcional al grado de Burnout (Bronfenbrenner, 1986). De cada una de las subescalas se obtuvieron medidas de tendencia central, así como estadísticos de dispersión. No se establecieron puntos de corte para estimar dada la heterogeneidad reportada en diversos estudios al momento de determinar prevalencias (Juárez et al., 2014). 
Además, también se indagó por características sociodemográficas y laborales del personal docente que labora en la institución educativa. En el análisis estadístico se utilizó el programa estadístico SPSS versión $20 \AA$, se calcularon frecuencias absolutas y relativas, medias y desviaciones estándar. Se utilizó la prueba no paramétrica de la mediana con el fin de estimar asociaciones entre variables. Los docentes diligenciaron en forma individual y anónima el cuestionario en su lugar de trabajo, bajo la supervisión de una persona adscrita a la investigación, quien previamente les explicó los objetivos del estudio, solicitó la participación voluntaria y responder lo más honestamente posible.

\section{RESULTADOS}

Los resultados se presentan en dos subsecciones: se definen y evalúan las características socios demográficos y laborales y luego se discute y evalúa el burnout en docentes participantes del estudio.

\section{Características socio demográficas y laborales}

En la Tabla 1, se observan las características sociodemográficas y laborales de los docentes encuestados. Los promedios de edad y antigüedad en el trabajo son de 41 y 6 años respectivamente. Aproximadamente la tercera parte de los docentes son hombres, $73,3 \%$ de los docentes encuestados tienen pareja. $40,0 \%$ de los docentes cuentan con contratos a término indefinido. 16,7\% de los encuestados tiene formación de postgrado. La mayor proporción de docentes encuestados (63.3\%) laboran en ciencias básicas, con cargas académicas definidas desde el principio del semestre.

Tabla 1: Sexo, estado civil, tipo de contrato, formación y área laboral de los docentes del estudio

\begin{tabular}{|c|c|c|}
\hline Genero & $N$ & $\%$ \\
\hline Femenino & 110 & 73,3 \\
\hline Masculino & 40 & 26,7 \\
\hline Estado Civil & $N$ & $\%$ \\
\hline Casado & 90 & 60,0 \\
\hline Divorciado & 20 & 13,3 \\
\hline Soltero & 20 & 13,3 \\
\hline Unión Libre & 20 & 13,3 \\
\hline Tipo de Contrato & $N$ & $\%$ \\
\hline Termino Fijo & 90 & 60,0 \\
\hline Término Indefinido & 60 & 40,0 \\
\hline Formación & $N$ & $\%$ \\
\hline Posgrado & 25 & 16,7 \\
\hline Especialista & 125 & 83,3 \\
\hline Área & $N$ & $\%$ \\
\hline Ciencias Básicas & 95 & 63,3 \\
\hline Ciencias de Énfasis & 55 & 36,7 \\
\hline Total & 150 & 100 \\
\hline
\end{tabular}

\section{Burnout en docentes participantes del estudio}

De un puntaje máximo posible de 54 en la escala de agotamiento emocional el promedio resultante fue de 17,1. En la escala de despersonalización de un máximo posible de 30 el puntaje promedio obtenido fue 6,3 . En cuanto a la escala de realización personal el puntaje medio fue de 40,3 de un máximo posible de 48 . El puntaje promedio de Burnout fue de 43,4 como cifra significativa de afectación entre los evaluados.

Tabla 2: Agotamiento Emocional, Despersonalización, Realización Personal y Burnout en docentes participantes

\begin{tabular}{lcccc}
\hline & Promedio & Desv. Estándar & Mediana & Rango Intercuartilico \\
\hline EE & 17,1 & 10,7 & 18 & $9-25$ \\
D & 6,3 & 5,5 & 4 & $2-11$ \\
PA & 40,3 & 5,5 & 42 & $37-44$ \\
Burnout & 43,4 & 3 & 44 & $40-45$ \\
\hline
\end{tabular}

La realización personal y el síndrome de Burnout se encuentran asociados al sexo al cual pertenecen los docentes siendo más alto ambos en el sexo femenino. La formación profesional resulta asociarse al agotamiento emocional puesto que los docentes que cuentan con solo pregrado reportan mayores puntajes 
en esta sub-escala. Similar hallazgo se obtiene en relación a la antigüedad laboral puesto que los de menor antigüedad presentan mayor agotamiento emocional, despersonalización y por ende Burnout, además se asocian al anterior fenómeno la baja realización personal y el agotamiento emocional que varía significativamente según las áreas entre los docentes encuestados. Los docentes que laboran en las áreas de ciencias básicas presentan menor agotamiento.

Tabla 3: Mediana de Agotamiento Emocional, Despersonalización, Realización Personal y Burnout según género, formación y antigüedad laboral en docentes participantes del estudio.

\begin{tabular}{lll}
\hline GENERO & Femenino & Masculino \\
\hline Mediana de EE & 20 & 16,5 \\
Mediana de D & 6,5 & 4 \\
Mediana de PA & 46 & 40 \\
Mediana de Burnout & 47,5 & 39 \\
\hline Formación & & \\
\hline & Especialización & Posgrado \\
\hline Mediana de EE & 21 & 16 \\
Mediana de D & 4 & 5 \\
Mediana de PA & 41 & 43 \\
Mediana de Burnout & 46 & 43 \\
\hline Antigüedad Laboral & & \\
\hline & Menos de 5 años & Más de 5 años \\
\hline Mediana de EE & 19 & 16 \\
Mediana de D & 4 & 2 \\
Mediana de PA & 39 & 43 \\
Mediana de Burnout & 45 & 39 \\
\hline
\end{tabular}

Son mayormente afectados por características clásicas del burnout las mujeres indistintamente de su nivel de especialización y antigüedad en relación con los hombres objeto del estudio, enfatizados en el agotamiento emocional al ser este el valor más preponderante entre los evaluados.

Tabla 4: Mediana de Agotamiento Emocional, Despersonalización, Realización Personal y Burnout según área laboral.

\begin{tabular}{|l|c|c|}
\cline { 2 - 3 } \multicolumn{1}{c|}{} & \multicolumn{2}{c|}{ AREA } \\
\cline { 2 - 3 } \multicolumn{1}{c|}{} & Ciencias Básicas & Ciencias de Énfasis \\
\hline Mediana de EE & 15 & 19 \\
\hline Mediana de D & 5 & 4 \\
\hline Mediana de PA & 42 & 43 \\
\hline Mediana de Burnout & 40 & 46 \\
\hline
\end{tabular}

Se observan valores positivos de realización personal entre los evaluados (positivos y negativos para burnout), aunque se podría inferir que este elemento no es decisivo para prevenir la aparición del síndrome. Por el contenido de las asignaturas, la mediana de afectados por el burnout fue superior entre los docentes que imparten la catedra de ciencias de énfasis para el caso puntual de este estudio.

\section{DISCUSIÓN}

La presencia de un alto nivel de Burnout entre los docentes participantes del presente estudio resulta menor a la encontrada por Oramas et al (2007), Díaz et al (2012) y Vilaret (2012) en países como Venezuela, Colombia y Ecuador respectivamente. La proporción de docentes con un alto nivel de Burnout es semejante a la hallada en México por Amador et al (2014), donde la prevalencia del síndrome fue del $22 \%$ del total de docentes encuestados, así como lo identificado por un estudio en docentes que laboran establecimientos educacionales municipalizados de la ciudad de Rengo (Chile), donde el 40,45\% de los participantes se encuentra afectado por Burnout (Jiménez et al., 2012). Estos datos ratifican que el síndrome de Burnout se ha convertido en un tema cada día más frecuente en el personal de docente, situación que debe despertar inquietudes pues sentirse "quemado" en el trabajo hace que el desempeño laboral se afecte, evidenciado en el aumento del ausentismo, incapacidades e incluso deserción laboral. 
Los resultados de esta investigación indican niveles altos de realización personal, similar al estudio hecho por Gantiva et al (2010), por lo que se esperaría que estos docentes tuvieran comportamientos relacionados con la comprensión, el diálogo, la claridad y la flexibilidad en cuanto al desarrollo de su trabajo; por el contrario, Padilla et al (2009), señala que los docentes presentan altos niveles en despersonalización y una baja realización personal. El agotamiento emocional constituye el componente de mayor afectación, reporta incluso valores mejores de asociación con el resto de las variables. A pesar de que el síndrome se caracteriza por la presencia de los tres componentes, el agotamiento emocional expresa mejor en los docentes el impacto de su trabajo en la salud física y mental. El nivel de agotamiento emocional identificado en el personal de docentes fue similar a los resultados obtenidos por Almeida et al (2015). Al comparar los resultados con un estudio realizado en docentes de Medellín-Colombia, la tendencia es igual puesto que la mayor parte de los encuestados se ubica en niveles medio y bajo de agotamiento emocional (Restrepo et al., 2006).

Al igual que otros estudios (Martínez, 2015; Díaz y Gómez, 2016) las mujeres reportan un mayor agotamiento emocional, a pesar de poseer una mayor realización personal en contraste con Oramas et al (2007). El agotamiento emocional resultó asociarse a la antigüedad laboral de manera que aquellos con mayor experiencia se agotan menos. En estudios como el de Fernández et al (2015), la antigüedad laboral no se asocia con el agotamiento emocional.

A diferencia de (Rojas et al., 2009), quienes en su estudio evidenció puntajes altos y significativos para agotamiento emocional, despersonalización y realización personal en el orden de $36.8 ; 33.4$ y 5,4\% respectivamente, en el actual estudio los valores difirieron con niveles inferiores, aunque igualmente confirmatorios para burnout. Contrastando con estudios realizados por Vysotskaya et al., (2015) y Cazan y Năstasă (2015) en cuyo estudio el síndrome se logró evidenciar por malestares de desgaste y disminución de la eficiencia en el trabajo, mientras que en la presente investigación no se hallaron tales situaciones, pero si coincide con las apreciaciones de Barutçu y Serinkan (2013) que determinaron afectaciones entre docentes donde la Inteligencia emocional es un insumo útil para sobreponerse a las dificultades que propina el síndrome y tiende a ser una de las recomendaciones principales de este estudio que hace énfasis en la manera en que podría hacerse frente a las complicaciones que el síndrome genera. Por distintas apreciaciones el proyecto de Košir et al., (2015) no predijo el estrés, sin embargo, identificó relaciones entre las características percibidas del trabajo y el estrés al igual que las identificas en esta investigación al confrontar en la relación menor antigüedad, mayor agotamiento emocional y despersonalización entre los evaluados positivos para burnout, que para el caso de los niveles profesionales abordados por De Caroli y Sagone (2013) fueron también evidentes síntomas de agotamiento emocional entre las mujeres que ostentan título de especialistas con poca antigüedad en el puesto de trabajo.

\section{CONCLUSIONES}

Con base en los resultados encontrados se puede concluir que, el síndrome burnout solo se asocia al género al cual pertenecen los docentes indicando que las mujeres padecen más con esta enfermedad laboral. Es importante mencionar que se encontró un bajo porcentaje del síndrome de burnout reflejadas en dos de sus tres dimensiones (agotamiento emocional y despersonalización) en los docentes, para la dimensión de falta de realización personal, se encontraron resultados altos asociados en gran medida a docentes que inician su actividad laboral. Vale la pena entonces recalcar que a medida que se alcanza experiencia el docente tiende a adaptase a su ejercicio laboral buscando estrategas didácticas que lo ayudan a tener menor agotamiento.

Definitivamente la formación profesional, mayor antigüedad y desarrollo de actividades en ciencias de énfasis, para este caso particular, logra asociarse con el agotamiento emocional y se ha establecido una concordancia entre docentes con solo pregrado, menor antigüedad y una mayor presencia de Burnout. Además, se asocian al anterior fenómeno la baja realización personal y el agotamiento emocional en los aquí citados.

\section{REFERENCIAS}

Almeida, M., Oliveira, E., Guimarães, F., Evangelista, L., Gomes, N. y Vieira, B. Síndrome de Burnout: un estudio con profesores. Salud de los Trabajadores, 23(1), 19-28 (2015)

Amador, R., Rodríguez, C., Serrano, J., Olvera, J., Martínez, I. y Ávila, S. Estrés y burnout en docentes de educación media superior. Revista Electrónica Medicina, Salud y Sociedad, 4(2), 119-141 (2014)

Arquero, J. y Donoso, J. Docencia, investigación y burnout: el síndrome del quemado en profesores universitarios de Contabilidad. Revista de Contabilidad, 16(2), 94-105 (2013) 
Barutçu, E. y Serinkan, C. Burnout Syndrome of Teachers: An Empirical Study in Denizli in Turkey. Procedia - Social and Behavioral Sciences, 89, 318-322 (2013)

Borges, A., Ruiz, M., Rangel, R. y González, P. Síndrome de Burnout en docentes de una universidad pública venezolana. Comunidad y Salud, 10(1), 1-9 (2012)

Botero, C. Riesgo psicosocial intralaboral y "burnout" en docentes universitarios de algunos países latinoamericanos. Revista Cuadernos de Administración, 28(48), 118-133 (2012)

Bronfenbrenner, W. The Ecology of Human Development. Harvard University Press. En versión castellana: Ecología del Desarrollo Humano. Barcelona: Paidós (1986)

Cazan, A. y Năstasă, L. Emotional Intelligence, Satisfaction with Life and Burnout among University Students, Procedia - Social and Behavioral Sciences, 180, 1574-1578 (2015)

De Caroli, M. y Sagone, E. Professional Self Representation and Risk of Burnout in School Teachers, Procedia - Social and Behavioral Sciences, 46, 5509-5515 (2012)

Díaz, F. y Gómez, I. La investigación sobre el síndrome de burnout en Latinoamérica entre 2000 y el 2010. Psicología desde El Caribe, 33(1), 113-131 (2016)

Díaz, F., López, A. y Varela, M. Factores asociados al síndrome de burnout en docentes de colegios de la ciudad de Cali, Colombia. Universitas Psychologica, 11(1), 217-227 (2012)

Fernández, V., Longás, J., Chamarro, A. y Virgili. C. Evaluando la salud laboral de los docentes de centros concertados: el Cuestionario de Salud Docente. Revista de Psicología del Trabajo y de las Organizaciones, 31(3), 175-185 (2015)

Fiorilli, C., Gabola, P., Pepe, A., Meylan, N., Curchod, D., Albanese, O. y Doudin, A. The effect of teachers' emotional intensity and social support on burnout syndrome. A comparison between Italy and Switzerland. Revue Européenne de Psychologie Appliquée/European Review of Applied Psychology, 65(6), 275-283 (2015)

Foley, C. y Murphy, C. Burnout in Irish teachers: Investigating the role of individual differences, work environment and coping factors. Teaching and Teacher Education, 50, 46-55 (2015)

Gantiva, C., Jaimes, S. y Villa, M. Síndrome de Burnout y estrategias de afrontamiento en docentes de primaria y bachillerato. Psicología desde EI Caribe, 26, 36-50 (2010)

Hozo, E., Sucic, G. y Zaja, I. Burnout Syndrome Among Educators in Pre-School Institutions. Material SocioMedica, 27(6), 399-403 (2015)

Jiménez, A., Jara, M. y Celis, E. Burnout, apoyo social y satisfacción laboral en docentes. Psicología Escolar e Educacional, 16(1), 125-134 (2012)

Juárez, A., Idrovo, A., Camacho, A., Placencia, O. Síndrome de burnout en población mexicana: Una revisión sistemática. Salud Mental, 37, 159-176 (2014)

Khezerlou, E. Teacher Self-efficacy as a Predictor of Job Burnout Among Iranian and Turkish EFL Teachers, Procedia - Social and Behavioral Sciences, 70, 1186-1194 (2013)

Košir, K., Tement, S., Licardo, M. y Habe, K. Two sides of the same coin? The role of rumination and reflection in elementary school teachers' classroom stress and burnout. Teaching and Teacher Education, 47, 131-141 (2015)

Kuimova, M.V., Uzunboylu, H., Chen, A.S.M., Gerasimchuk, E.V. Emotional burnout in professional activity of a technical university teacher. Ponte, Florence, Italy. Inter. Scientific Researches Journal, 72 (6) 57-61, junio (2016)

Malinen, O. y Savolainen, H. The effect of perceived school climate and teacher efficacy in behavior management on job satisfaction and burnout: A longitudinal study. Teaching and Teacher Education, 60, 144$152(2016)$

Martínez, J. Cómo se defiende el profesorado de secundaria del estrés: burnout y estrategias de afrontamiento. Revista de Psicología del Trabajo y de las Organizaciones, 31(1), 1-9 (2015) 
Maslach, C. y Jackson, S. Maslach Burnout Inventory. Research Edition. Palo Alto, California: Consulting Psychologists Press, 2, 99-113 (1981)

Mojsa, J., Golonka, K. y Marek, T. Job burnout and engagement among teachers - Worklife areas and personality traits as predictors of relationships with work. International Journal Of Occupational Medicine \& Environmental Health, 28(1), 102-119 (2015)

Oramas, A., Almirall, P. y Fernández, I. Estrés Laboral y el Síndrome de Burnout en Docentes Venezolanos. Salud de los Trabajadores, 15(2), 71-87 (2007)

Padilla, A., Gómez, C., Rodríguez, V., Dávila, M., Avella, C., Caballero, A. y Vives, A. Prevalencia y características del síndrome de agotamiento profesional (SAP) en docentes de tres colegios públicos de Bogotá (Colombia). Revista Colombiana de Psiquiatría, 38, 50- 65 (2009)

Restrepo, N., Colorado, G. y Cabrera, G. Desgaste Emocional en Docentes Oficiales de Medellín, Colombia, 2005. Rev. Salud Pública, 8(1), 63-73 (2006)

Rojas, M., Zapata, J. y Grisales, H. Síndrome de burnout y satisfacción laboral en docentes de una institución de educación superior, Medellín. Rev. Fac. Nac. Salud Pública, 27(2), 198-210 (2009)

Suárez, A. Subjective Well-being (Sb) and Burnout Syndrome (BnS): Correlational Analysis Teleworkers Education Sector, Procedia - Social and Behavioral Sciences, 237, 1012-1018 (2017)

Vilaret, A. Carga mental y Síndrome de Burnout en docentes a tiempo completo de una universidad de Quito. Revista Eidos, 6, 36-47 (2012)

Vysotskaya, N., Cherkashina, E., Katcin, O. y Lisina, L. Studies on University Professors' Emotional Burnout, Procedia - Social and Behavioral Sciences, 214, 769-778 (2015) 